\title{
Пространственное распределение шаровых скоплений в Галактике
}

\author{
Н.P. Аракелян ${ }^{1,2}$, С.В. Пилипенко, М.Е. Шарина ${ }^{3}$ \\ 1 Московский физико-технический институт (государственный университет), Институтский пер., 9, \\ Долгопрудный, Россия, 141701 \\ n.rubenovna@mail.ru \\ 2 Физический институт им. П.Н. Лебедева РАН, Профсоюзная, 84/32, Москва, Россия, 117997 \\ spilipenko@asc.rssi.ru \\ 3 Специальная астрофизическая обсерватория РАН, Нижний Архыз, Россия, 369167 \\ sme@sao.ru
}

Поступила в редакцию 3 ноября 2017 г.

\begin{abstract}
Аннотация. В статье проведены подсчеты для выявления вероятных плоскостей в системах шаровых скоплений и галактик-спутников нашей Галактики и оценена статистическая достоверность результатов двумя разными методами, а также проведен поиск шаровых скоплений, принадлежащих к приливным потокам Стрельца и Единорога, которые образовались в результате частичных разрушений спутников. Была измерена анизотропия распределения шаровых скоплений без приливных потоков и в области влияния потоков. Измерения показали, что для шаровых скоплений и галактикспутников, ограниченных максимальным расстоянием выборки от центра Галактики, наблюдается некоторая анизотропия, но исключение составляет $\mathrm{R}<18$ кпк для шаровых скоплений. Измерение анизотропии распределения шаровых скоплений в Млечном Пути без приливных потоков показало, что потоки вносят небольшой вклад в анизотропию распределения шаровых скоплений.
\end{abstract}

SPATIAL DISTRIBUTION OF GLOBULAR CLUSTERS IN THE GALAXY, by N.R. Arakelyan, S.V. Pilipenko, M.E. Sharina. In this paper, we performed calculations to identify probable planes in the systems of globular clusters and satellite galaxies of our Galaxy and estimated the statistical significance of the results by two different methods, and also searched for globular clusters belonging to tidal streams of Sagittarius and Monoceros that were formed as a result of partial destruction of satellites. We measured the anisotropy of the distribution of globular clusters without tidal streams and in the region of the influence of streams. Measurements showed that for globular clusters and satellite galaxies limited by the maximum sampling distance from the center of the Galaxy, some anisotropy is observed, but the exception is $\mathrm{R}<18 \mathrm{kpc}$ for globular clusters. Measurement of the anisotropy of the distribution of globular clusters in the Milky Way without tidal streams has shown that the streams contribute little to the anisotropy of the distribution of globular clusters.

Ключевые слова: Галактика: структура; шаровые скопления 


\section{1 Введение}

Некоторые шаровые скопления (ШС), принадлежащие Млечному Пути, родились не в самой нашей Галактике, а попали туда из-за приливных потоков, которые образовались в результате частичных разрушений галактик-спутников. Чтобы проверить, как влияет аккреция галактик-спутников на распределение ШС, мы планируем: измерить анизотропию распределения ШС и галактик-спутников; проверить достоверность результатов; определить принадлежность ШС приливным потокам; измерить анизотропию распределения ШС без приливных потоков.

\section{2 Измерение анизотропии}

В нашей Галактике количество ШС составляет 157 (Харрис и др., 2013), а количество галактикспутников, принадлежащих нашей Галактике, составляет 27 (МакКоннакей, 2012).

Для проверки влияния аккреции галактик-спутников на распределение ШС нужно измерить анизотропию распределения ШС и галактик-спутников. В этой работе анизотропия распределения была проверена с помощью тензора собственных размеров (gyration tensor), который строится аналогично тензору инерции:

$$
S_{i j}=\frac{1}{N} \sum_{k=1}^{N} x_{i}^{k} x_{j}^{k},
$$

где $\mathrm{S}$ - тензор размеров; $\mathrm{N}$ - количество объектов; х - расстояния от точек до центра Галактики.

Для проверки статистической значимости было использовано два разных метода. Для них было сгенерировано 10000 выборок со случайными координатами объектов. Разница между методами I и II заключается в построении случайных каталогов. Для метода I мы берем куб со стороной 2R, помещаем туда объекты: случайно и равномерно, из этого куба вырезаем сферу с радиусом $\mathrm{R}$, где $\mathrm{R}-$ максимальное расстояние выборки от центра Галактики. В сферу попадает столько объектов, сколько наблюдается ШС до расстояния R. Для метода II для каждого объекта мы берем расстояния (R) из реального каталога, а галактическую широту (b) и долготу (l) даем случайным образом.

Мы разделили наш каталог на выборки по расстоянию от центра Галактики. И для каждой выборки измерили анизотропию распределения. В таблице 1 представлены результаты.

В качестве критерия статистической значимости мы использовали:

$$
|\max / \min -\langle\max / \min \rangle|>3 \sigma
$$

где $\mathrm{max} / \mathrm{min}$ - максимальное собственное значение, разделенное на минимальное собственное значение; $\langle\max / \min \rangle$ - среднее значение $\max / \min$ для случайного каталога.

Полученные данные показывают, что для метода I для всех выборок ШС и галактик-спутников наблюдается статистически значимая анизотропия. Но исключение составляет $\mathrm{R}<18$ кпк для ШС. Для метода II для ШС получается статистически значимая анизотропия только на расстояниях 5 и 10 кпк, а для галактик-спутников - на расстояниях 258 и 189 кпк.

Некоторые ШС родились не в самой нашей Галактике, а попали туда из приливных хвостов потоков. Представлял интерес поиск ШС, которые принадлежат этим приливным потокам. Для проверки были выбраны два потока: поток Стрельца (Sagittarius) (Карбалло-Белло и др., 2014) и поток Единорога (Monoceros) (Пенаррубия и др., 2005). Выводы о принадлежности были сделаны на основе трех критериев: положение на небе; радиальная скорость; расстояние от Солнца. Количество найденных новых ШС в потоке Стрельца составляет 30, а в потоке Единорога - 12 .

Для того чтобы понять, влияют ли потоки на распределение ШС в нашей Галактике, была измерена анизотропия распределения для всех ШС: 1) без потока Стрельца; 2) без потока Единорога; 3) без потока Стрельца и Единорога одновременно. Результаты показывают, что для метода I проверки достоверности для всех выборок получается статистически значимая анизотропия. А для метода II для всех ШС без потоков (все 3 случая) не наблюдается статистически значимая анизотропия. 
Таблица 1. Результаты для тензора размеров

\begin{tabular}{|c|c|c|c|c|c|c|c|c|c|c|c|c|}
\hline & $\mathrm{R}^{1)}$ & $\mathrm{N}^{2)}$ & $W_{1}{ }^{3)}$ & $W_{2}{ }^{3)}$ & $W_{3}{ }^{3)}$ & $\max / \min { }^{4)}$ & $\begin{array}{c}\langle\max / \min \rangle^{5)} \\
\text { Метод I }\end{array}$ & $\begin{array}{c}\langle\max / \min \rangle^{5)} \\
\text { Метод II }\end{array}$ & $\sigma_{1}{ }^{6)}$ & $\sigma_{2}{ }^{6)}$ & $\mathrm{I}^{7)}$ & II $^{7)}$ \\
\hline \multirow[t]{8}{*}{ ШС } & $<125$ & 157 & 8.819 & 14.522 & 16.059 & 1.821 & 1.15 & 1.715 & 0.053 & 0.284 & $13 \sigma$ & $0.4 \sigma$ \\
\hline & $<100$ & 155 & 7.725 & 13.362 & 11.62 & 1.729 & 1.149 & 1.668 & 0.052 & 0.245 & $11 \sigma$ & $0.2 \sigma$ \\
\hline & $<75$ & 152 & 6.404 & 9.693 & 8.479 & 1.513 & 1.151 & 1.466 & 0.054 & 0.157 & $7 \sigma$ & $0.3 \sigma$ \\
\hline & $<50$ & 151 & 6.063 & 8.053 & 8.537 & 1.408 & 1.152 & 1.379 & 0.054 & 0.139 & $5 \sigma$ & $0.2 \sigma$ \\
\hline & $<24$ & 138 & 5.754 & 4.973 & 4.248 & 1.355 & 1.16 & 1.291 & 0.057 & 0.109 & $3 \sigma$ & $0.6 \sigma$ \\
\hline & $<18$ & 128 & 4.674 & 3.739 & 4.055 & 1.25 & 1.167 & 1.297 & 0.059 & 0.111 & $\sigma$ & $0.4 \sigma$ \\
\hline & $<10$ & 106 & 3.19 & 1.884 & 2.737 & 1.693 & 1.185 & 1.272 & 0.067 & 0.099 & $8 \sigma$ & $4 \sigma$ \\
\hline & $<5$ & 74 & 2.141 & 1.482 & 1.261 & 1.697 & 1.226 & 1.279 & 0.083 & 0.104 & $6 \sigma$ & $4 \sigma$ \\
\hline \multirow[t]{5}{*}{$\overline{\Gamma-C}$} & $<258$ & 27 & 104.227 & 28.699 & $\overline{53.709}$ & 3.632 & 1.422 & 18.731 & 0.178 & 0.312 & $12 \sigma$ & $\overline{26 \sigma}$ \\
\hline & $<189$ & 24 & 26.726 & 49.564 & 78.498 & 2.937 & 1.462 & 1.725 & 0.201 & 0.313 & $7 \sigma$ & $4 \sigma$ \\
\hline & $<150$ & 20 & 60.668 & 33.396 & 26.045 & 2.329 & 1.522 & 1.791 & 0.243 & 0.335 & $3 \sigma$ & $2 \sigma$ \\
\hline & $<121$ & 18 & 50.05 & 20.029 & 32.487 & 2.499 & 1.569 & 1.747 & 0.265 & 0.338 & $4 \sigma$ & $2 \sigma$ \\
\hline & $<80$ & 14 & 42.109 & 15.87 & 20.477 & 2.653 & 1.689 & 1.844 & 0.369 & 0.397 & $3 \sigma$ & $2 \sigma$ \\
\hline
\end{tabular}

1) $\mathrm{R}$ - максимальное расстояние выборки от центра Галактики в кпк.

2) $\mathrm{N}$ - количество объектов.

3) W1, W2, W3 - собственные значения тензора.

4) $\mathrm{max} / \mathrm{min}$ - максимальное собственное значение, разделенное на минимальное собственное значение.

5) $\langle\max / \min \rangle$ метод I и II - среднее значение $\max / \min$ для случайного каталога (для методов I и II).

6) $(\sigma 1,2)$ - дисперсия для случайного каталога (для методов I и II).

7) I, II - статистическая значимость для методов I и II.

Приливные потоки лежат на расстоянии $1 \div 39$ кпк от центра Галактики. Результаты проверки анизотропии в этой области показывают, что для метода I получается статистическая значимая анизотропия, но если убрать потоки, то анизотропия пропадает. А для метода II, с/без потоков, статистически значимой анизотропии нет, и удаление потоков общую картину не меняет.

\section{3 Обсуждение и заключение}

Измерения отношений собственных значений тензора показали, что для всех выборок ШС и галактикспутников, ограниченных максимальным расстоянием выборки от центра Галактики, наблюдается некоторая анизотропия. Исключение составляет $\mathrm{R}<18$ кпк для ШС.

В этой статье были рассмотрены потоки Стрельца и Единорога. Была измерена анизотропия распределения ШС с/без потоков для всего разброса ШС и в области влияния потоков $(1 \div 39$ кпк). Измерение анизотропии распределения ШС без приливных потоков показало, что потоки вносят небольшой вклад в анизотропию распределения ШС.

\section{Литература}

Карбалло-Белло и др. (Carballo-Bello J.A., Sollima A., Martinez-Delgado D., Pila-Diez B., Leaman R., et al.) // Mon. Not. Roy. Astron. Soc. 2014. V. 445. P. 2971.

МакКоннакей (McConnachie A.W.) // Astron. J. 2012. V. 144. P. 4.

Пенаррубия и др. (Penarrubia J., Martinez-Delgado D., Rix H.W., Gomez-Flechoso M.A., Munn J., et al.) // Astrophys. J. 2005. V. 626. P. 128.

Харрис и др. (Harris W.E., Harris G.L.H., Alessi M.) // Astrophys. J. 2013. V. 772. P. 82. 